\title{
AVALIAÇÃO DO MÓDULO DE ELASTICIDADE DA MADEIRA COM USO DE MÉTODO NÃO-DESTRUTIVO DE VIBRAÇÃO TRANSVERSAL
}

\author{
LUIZ A. TARGA ${ }^{1}$, ADRIANO W. BALLARIN ${ }^{2}$, MARCO A. M. BIAGGIONI ${ }^{3}$
}

RESUMO: Numerosas pesquisas têm estudado os métodos não-destrutivos de avaliação de materiais e sua aplicação àqueles de matrizes complexas, como é o caso da madeira. Um dos primeiros métodos não-destrutivos investigados para aplicação nesses casos foi o da vibração transversal. Apesar de sua concepção simples, e a despeito dos grandes avanços obtidos nessa área com outros métodos, como, por exemplo, o ultra-som, o método de vibração transversal para a determinação do módulo de elasticidade da madeira revela-se como de grande potencial de aplicação, sobretudo pela precisão do modelo matemático a ele associado e pela possibilidade de sua aplicação a peças de dimensões estruturais (in-grade testing). Neste trabalho, apresenta-se o uso desse método na determinação do módulo de elasticidade de três espécies de eucalipto. Foram ensaiados não-destrutivamente e por ensaios mecânicos convencionais de flexão corpos-de-prova de $2 \mathrm{~cm}$ x $2 \mathrm{~cm}$ x $46 \mathrm{~cm}$ de $E$. grandis, $E$. saligna e E. citriodora. Os ensaios não-destrutivos foram conduzidos com uso do sistema BING Beam Identification by Non-destructive Grading, que permite a análise das vibrações do material nos domínios do tempo e da freqüência. Os resultados obtidos revelaram boa correlação entre os dois tipos de ensaios empregados, justificando o início dos ensaios com peças de dimensões estruturais, para a viabilização da técnica nas práticas de classificação estrutural.

PALAVRAS-CHAVE: ensaios não-destrutivos, vibração transversal, Eucalyptus sp.

\section{EVALUATION OF WOOD ELASTICITY MODULUS BY NON-DESTRUCTIVE RESONANCE METHOD OF TRANSVERSAL VIBRATION}

\begin{abstract}
Numerous researches have been developed, applying non-destructive techniques to complex matrix materials, e.g. wood. The resonance method was one of the first studied for the determination of MOE of wood. Despite the great advances obtained in this matter, with the development of special techniques like ultrasound, the resonance method reveals a great potential of utilization mainly due to the well defined mathematical model associated to the physical phenomenon and the possibility to use it on in-grade test (using commercial size lumbers). This paper presents the application of this non-destructive method to three species of eucalyptus. Using resonance nondestructive method and posterior bending static tests were analyzed specimens of $2 \mathrm{~cm} \times 2 \mathrm{~cm}$ x $46 \mathrm{~cm}$ of E. grandis, E. saligna e E. citriodora. Non-destructive tests were carried out using BING - Beam Identification by Non-destructive Grading, a commercial system that analyses vibrations in the time and frequency domains. Results revealed a great correlation between MOE determined by the nondestructive and the conventional methods, justifying the beginning of an experimental program with commercial size lumbers.
\end{abstract}

KEYWORDS: non-destructive tests, transverse vibration, Eucalyptus $s p$.

\footnotetext{
${ }^{1}$ Prof. Titular, Departamento de Engenharia Rural, UNESP - Câmpus de Botucatu - SP, targa@ fca.unesp.br

${ }^{2}$ Prof. Adjunto, Departamento de Engenharia Rural, UNESP - Câmpus de Botucatu - SP, awballarin@ fca.unesp.br

${ }^{3}$ Prof. Assistente Doutor, Departamento de Engenharia Rural, UNESP - Câmpus de Botucatu - SP, biaggioni@ fca.unesp.br

Recebido pelo Conselho Editorial em: 28-11-2003

Aprovado pelo Conselho Editorial em: 7-6-2005
}

Eng. Agríc., Jaboticabal, v.25, n.2, p.291-299, maio/ago. 2005 


\section{INTRODUÇÃO}

Segundo a ABENDE (2002), os ensaios não-destrutivos são aqueles realizados em materiais para verificar a existência ou não de descontinuidades ou defeitos, por meio de princípios físicos definidos, sem alterar suas características físicas, químicas, mecânicas ou dimensionais e sem interferir em seu uso posterior.

Os métodos mais usuais utilizam-se de ultra-som, radiografia, correntes parasitas, análise de vibrações, emissão acústica, entre outros. Na área de pesquisas, as avaliações não-destrutivas, utilizando vibrações e ultra-som, são empregadas para a determinação das propriedades físicas e mecânicas de materiais. Para alguns materiais, como os metais e ligas metálicas, essa técnica tem sido bastante usada, uma vez que pode detectar defeitos e descontinuidade nesses materiais. No caso da madeira, como esses problemas são característicos do material, os ensaios não-destrutivos são usados, quase sempre, para verificar como essas ocorrências afetam as suas características físicas e mecânicas.

As primeiras pesquisas relativas à aplicação de ensaios não-destrutivos na determinação das propriedades físicas e mecânicas da madeira foram realizadas na década de 1950, nos Estados Unidos. Ainda que detenham a primazia de conhecimentos nessa área específica, alguns países como Suíça, Romênia, Alemanha, Japão e Reino Unido, entre outros, também já se destacam nesse cenário. Embora seja grande a variedade de técnicas não-destrutivas para avaliação da madeira, algumas delas, citadas por ROSS \& PELLERIN (1994), têm sido objeto de maior investigação e uso por parte dos institutos de pesquisa. Como exemplos, podem-se citar:

- Técnica da flexão estática - consiste na medição estática do MOE - Módulo de elasticidade na flexão de um exemplar de madeira, sendo o princípio básico de funcionamento da máquina de classificação da madeira serrada. MSR - Machine Stress Rating;

- Técnica da vibração transversal (transverse vibration technique or ressonance technique) consiste na análise da vibração de um exemplar de madeira, com obtenção do módulo de elasticidade do material a partir da análise das freqüências principais de vibração;

- Técnica das ondas de tensão (stress wave method) - consiste na aplicação de uma onda de tensão (impacto) no material e análise do fenômeno de propagação desse estímulo. A velocidade de propagação de uma onda de tensão induzida e sua atenuação no material são os principais parâmetros analisados nesses casos, e

- Técnica da propagação de ondas de ultra-som - diversos institutos de pesquisa têm voltado suas atenções para as técnicas de ondas acústicas (Acoustic emission - AE) e ultra-sônicas (Acoustoultrasonic emission - $A U$ ), que se têm mostrado eficientes para determinados propósitos, como, por exemplo, a avaliação da orientação da grã da madeira.

Este trabalho apresenta resultados preliminares de estudo encaminhado junto ao Laboratório de Ensaio de Materiais - FCA/UNESP, para análise das potencialidades de aplicação do método da vibração transversal na obtenção de parâmetros elásticos de algumas madeiras de reflorestamento do Brasil.

\section{Técnica não-destrutiva da vibração transversal}

Comumente referida como "resonance method" na bibliografia internacional, essa técnica temse destacado entre as empregadas em testes não-destrutivos, sobretudo pela grande aderência entre o modelo físico do fenômeno e o correspondente modelo matemático teórico. Além disso, a dificuldade de mensuração das altas freqüências observadas em outros métodos não-destrutivos - o método do ultra-som, por exemplo, freqüentemente se utiliza de transmissões de ondas a $100 \mathrm{kHz}$ ou $150 \mathrm{kHz}$ faz com que o método da ressonância se torne ainda mais interessante. $\mathrm{O}$ equacionamento teórico da vibração em barras e placas, baseado no modelo de Euler e Saint-Venant, é apresentado em diversos 
livros, como HEARMON (1961), SNOWDON (1968), GOUGH et al. (1983), BODIG \& JAYNE (1993) e BUCUR (1995), resultando:

$$
\mathrm{EI} \frac{\partial^{4} \mathrm{v}}{\partial \mathrm{x}^{4}}+\rho \mathrm{A} \frac{\partial \mathrm{v}^{2}}{\partial \mathrm{t}^{2}}=0
$$

em que,

E - módulo de elasticidade;

I - momento de inércia;

$\mathrm{v}$ - deslocamento na direção y ou z, normais ao eixo da viga;

$\mathrm{x}$ - distância ao longo do eixo da viga;

$\rho$ - densidade de massa;

A - área da seção transversal da viga, e

$\mathrm{t}$ - tempo.

A solução geral para a eq.(1), considerando-se a vibração transversal de barras com extremidades livres (free-free condition), é:

$$
E=\frac{4 \pi^{2} f_{n}{ }^{2} 1^{4} A \rho}{I m_{n}^{4}}
$$

em que,

$\mathrm{f}_{\mathrm{n}}$ - freqüência de vibração associada ao modo " $\mathrm{n}$ " (n-ésima harmônica ), $\mathrm{Hz}=\mathrm{s}^{-1}$;

1 - comprimento da barra, m;

$\mathrm{m}_{\mathrm{n}}$ - fator de correção associado à n-ésima harmônica, sem unidade, $\mathrm{e}$

$[\mathrm{E}]=\mathrm{Pa} ;[\mathrm{\rho}]=\mathrm{kg} \mathrm{m}^{-3} ;[\mathrm{A}]=\mathrm{m}^{2} ;[\mathrm{I}]=\mathrm{m}^{4}$

No geral, analisa-se a vibração transversal de barras monitorando-se sua freqüência de ressonância principal ( $1^{\underline{a}}$ harmônica), e, nesse caso, a eq.(2) toma a forma:

$$
E=E_{d}=\frac{0,9464 \rho f_{1}{ }^{2} 1^{4}}{h^{2}}
$$

em que,

$\mathrm{E}_{\mathrm{d}}$ - módulo de elasticidade dinâmico (o índice "d" diferencia esse módulo daquele por meio através de ensaios convencionais estáticos), $\mathrm{Pa}$, e

h - altura da barra, m.

Essa expressão simplificada tem sido utilizada pela grande maioria dos pesquisadores que empregam o método da ressonância (vibração transversal). Refinamentos teóricos à formulação básica foram propostos por PERSTORPER (1993), contabilizando-se as deformações por cisalhamento e torção e pelo CIRAD (1998). Pelas experiências nacionais no uso do método da ressonância, nota-se que as melhorias advindas do equacionamento mais refinado não são significativas para relações vãoaltura da peça maiores que 21 .

Com a eq.(3), pode-se empregar o método da ressonância para a determinação do módulo de elasticidade dinâmico de pequenas peças, em escala laboratorial, como também de peças de dimensões comercias e roliças (WANG et al., 2002) ou serradas (CALIL JÚNIOR \& MINÁ, 2003).

BURDZIK \& NKWERA (2002) propuseram que, com a expansão de domínio da informática, o método da ressonância pode ser uma simples e confiável alternativa para os métodos estáticos que necessitam de equipamentos caros e têm operação mais complexa. 
Não são reportadas na bibliografia interferências de ruídos, tanto na percussão quanto na aquisição dos sinais.

\section{MATERIAL E MÉTODOS}

Neste trabalho, são avaliados os módulos de elasticidade da madeira de E. grandis, E. saligna e E. citriodora, usando-se a técnica das vibrações transversais (transverse vibration technique). A madeira de E. citriodora era originária de plantios do Horto Florestal de Manduri. Foram amostradas 15 árvores com 31 anos de idade, de talhão com densidade populacional de 245 árvores por hectare.

As madeiras de E. saligna e E. grandis foram provenientes de plantios conduzidos pela empresa Eucatex, no município de Bofete - SP. Foram amostradas 12 árvores de E. saligna com idade de 26 anos, de talhão com 200 árvores por hectare. Para a caracterização da madeira de E. grandis, utilizaram-se 24 árvores com 21 anos de idade, de talhão com 200 árvores por hectare.

Para a produção dos corpos-de-prova foram utilizados os pranchões centrais das madeiras de $E$. citriodora, E. grandis e E. saligna. A madeira proveniente das três espécies de eucalipto foi climatizada em sala de climatização do Instituto de Pesquisas Tecnológicas - IPT, São Paulo, até atingir umidade de equilíbrio de aproximadamente $12 \%$.

Depois da climatização e secagem do material, a partir dos pranchões, foram confeccionados corpos-de-prova prismáticos, com dimensões nominais $2 \mathrm{~cm} \times 2 \mathrm{~cm}$ x $46 \mathrm{~cm}$, com as arestas das seções transversais coincidentes com as direções radial e tangencial da madeira. Amostraram-se, no mínimo, dois corpos-de-prova por árvore, provenientes do primeiro torete a partir da base da árvore.

Os corpos-de-prova foram ensaiados subseqüentemente para a determinação do módulo de elasticidade por dois processos distintos:

- Por meio de ensaio não-destrutivo de vibração transversal, com uso do equipamento BING, e

- Por meio de ensaio destrutivo de flexão estática, seguindo-se, no geral, as prescrições do anexo B da NBR 7190 (ABNT, 1997).

Para o ensaio de flexão, manteve-se a relação vão/altura do corpo-de-prova sugerida pelo código normativo, bem como as velocidades e etapas gerais de carregamento, considerando-se as pré-cargas que antecedem o ensaio rotineiro.

O equipamento utilizado para a análise e a mensuração das frequiências de vibração transversal foi o BING - Beam Identification by Nondestructive Grading, produzido pelo CIRAD-Foret - Centre de coopération internationale en recherche agronomique pour le développement - Département forestier. No procedimento de ensaio, a vibração é introduzida na amostra por meio de impacto mecânico (martelo). O sinal é captado com uso de microfone, sendo digitalizado, por meio de placa externa ao computador. O programa de computador executa a análise espectral, selecionando a frequiência própria de vibração da peça e contabilizando o módulo de elasticidade com o uso da eq.(3).

\section{RESULTADOS E DISCUSSÃO}

Na Tabela 1, apresenta-se o número de corpos-de-prova ensaiados para cada espécie de madeira e dados gerais a respeito da densidade aparente dessas madeiras, obtidos por meio da pesagem e mensuração dos corpos-de-prova de flexão $(2 \mathrm{~cm}$ x $2 \mathrm{~cm}$ x $46 \mathrm{~cm})$ e determinado na umidade de $12 \%$.

Os valores de densidade aparente média a 12\% de umidade resultaram bastante próximos daqueles sugeridos para as espécies pela NBR 7190 (ABNT, 1997). 
TABELA 1. Dados gerais sobre os corpos-de-prova das espécies ensaiadas.

\begin{tabular}{lccc}
\hline \multirow{2}{*}{ Variáveis } & \multicolumn{3}{c}{ Madeira } \\
\cline { 2 - 4 } & E. citriodora & E. grandis & E. saligna \\
\hline Número de c.p. & 69 & 57 & 28 \\
Densidade mínima $\left(\mathrm{g} \mathrm{cm}^{-3}\right)$ & 0,822 & 0,412 & 0,634 \\
Densidade máxima $\left(\mathrm{g} \mathrm{cm}^{-3}\right)$ & 1,170 & 0,872 & 1,017 \\
Densidade média $\left(\mathrm{g} \mathrm{cm}^{-3}\right)$ & 1,003 & 0,624 & 0,877 \\
Desvio-Padrão & 0,059 & 0,105 & 0,101 \\
Coeficiente de variação $(\%)$ & 5,88 & 16,79 & 11,52 \\
\hline
\end{tabular}

Na Tabela 2, detalham-se os valores obtidos para o módulo de elasticidade dinâmico $\left(\mathrm{E}_{\mathrm{d}}\right)$, com uso da técnica da vibração transversal (Figura 1).

TABELA 2. Valores gerais obtidos para o módulo de elasticidade dinâmico $\left(\mathrm{E}_{\mathrm{d}}\right)$.

\begin{tabular}{lccc}
\hline \multirow{2}{*}{ Modelo Elástico Dinâmico $\left(\mathrm{E}_{\mathrm{d}}\right)$} & \multicolumn{3}{c}{ Madeira } \\
\cline { 2 - 4 } & E. citriodora & E. grandis & E. saligna \\
\hline Valor mínimo $(\mathrm{MPa})$ & 10.448 & 12.149 & 16.547 \\
Valor máximo $(\mathrm{MPa})$ & 30.791 & 21.846 & 23.726 \\
Valor médio $(\mathrm{MPa})$ & 24.704 & 16.990 & 19.566 \\
Desvio-Padrão & 3.519 & 2.446 & 1.927 \\
Coeficiente de variação $(\%)$ & 14,24 & 14,40 & 9,85 \\
\hline
\end{tabular}

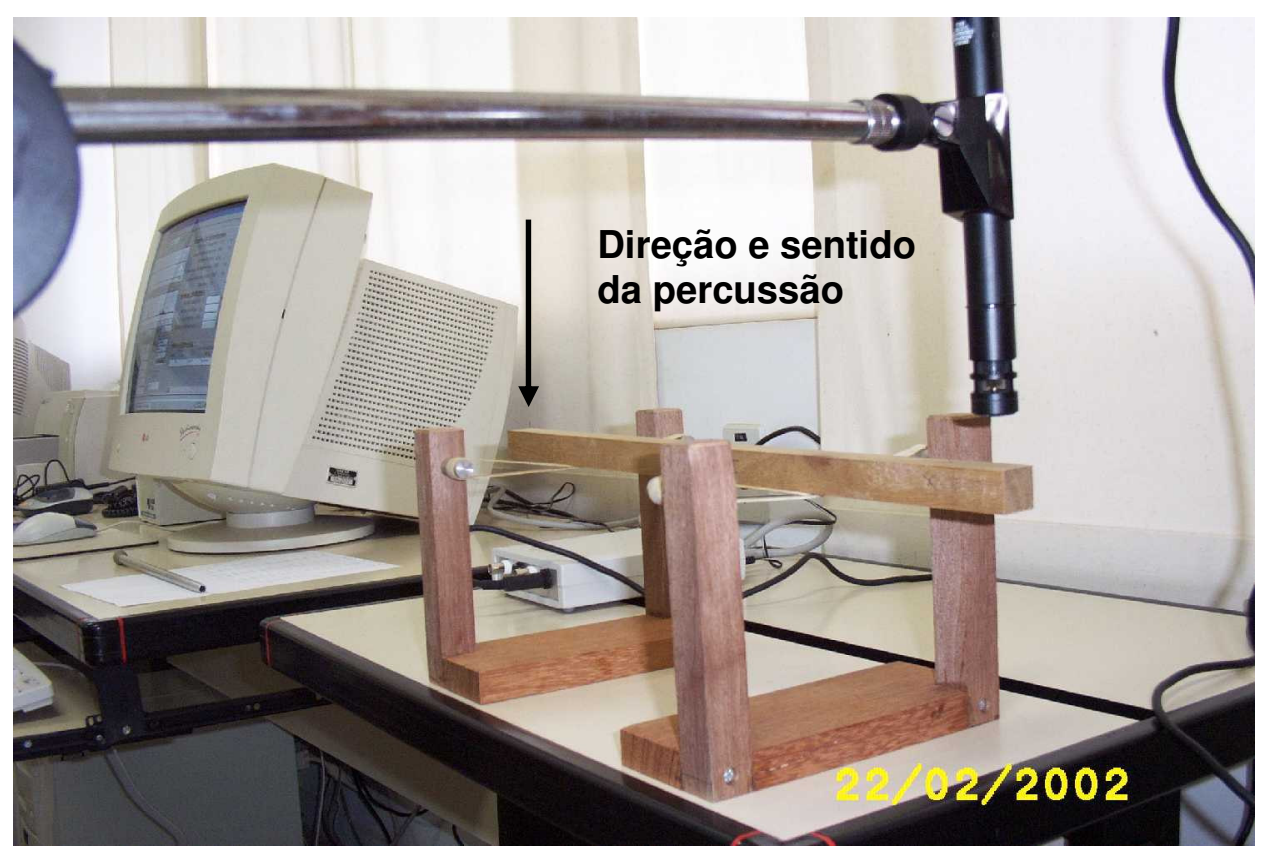

FIGURA 1. Aparato geral utilizado no ensaio de vibração transversal, com detalhe do posicionamento do corpo-de-prova apoiado nos "quartos" e o microfone unidirecional para a captação da vibração.

Na Tabela 3, resumem-se os valores do módulo de elasticidade à flexão da madeira, obtidos a partir de ensaios destrutivos convencionais, realizados seguindo-se, no geral, as prescrições da NBR7190 (ABNT, 1997). 
TABELA 3. Valores gerais obtidos para o módulo de elasticidade estático (E).

\begin{tabular}{lccc}
\hline \multirow{2}{*}{ Modelo Elástico Estático (E) } & \multicolumn{3}{c}{ Madeira } \\
\cline { 2 - 4 } & E. citriodora & E. grandis & E. saligna \\
\hline Valor mínimo (MPa) & 15.120 & 11.018 & 12.515 \\
Valor máximo (MPa) & 26.548 & 19.166 & 18.613 \\
Valor médio (MPa) & 22.420 & 15.306 & 15.285 \\
Desvio-Padrão & 2.196 & 2.152 & 1.498 \\
Coeficiente de variação (\%) & 9,79 & 14,06 & 9,80 \\
\hline
\end{tabular}

Os valores obtidos para o módulo de elasticidade estático das madeiras estudadas revelaram-se ligeiramente superiores àqueles apresentados na NBR 7190 (ABNT, 1997). Da análise conjunta das Tabelas 2 e 3, nota-se que os coeficientes de variação encontrados ficaram dentro dos limites aceitáveis para ensaios mecânicos (C.V. $\leq 20 \%)$ e revelaram, ainda, grande proximidade entre os valores para cada espécie, nos dois ensaios realizados.

Nas Figuras 2 a 4, ilustram-se as correlações obtidas entre os módulos de elasticidade dinâmico $\left(E_{d}\right)$ e estático $(E)$.

Os resultados gráficos apresentados revelaram a boa correlação existente entre os módulos de elasticidade dinâmicos e estáticos $\left(0,76 \leq \mathrm{R}^{2} \leq 0,87\right)$.

Para o E. saligna, o relativamente pequeno número de corpos-de-prova (todas as demais séries foram conduzidas com cerca 50 corpos-de-prova) pode ter comprometido o estabelecimento de uma correlação mais adequada.

Em todas as situações, conforme já observado por outros pesquisadores internacionais, o módulo de elasticidade dinâmico revelou-se maior que o estático $(\mathrm{Ed}>\mathrm{E})$. Estudos recentes dão conta de que, em função de sua natureza viscoelástica, o menor valor para o módulo de elasticidade da madeira é sempre aquele obtido com uso de ensaios estáticos. Daí em diante, os valores para os módulos de elasticidade são crescentes com o aumento da freqüência de excitação utilizada nos ensaios dinâmicos.

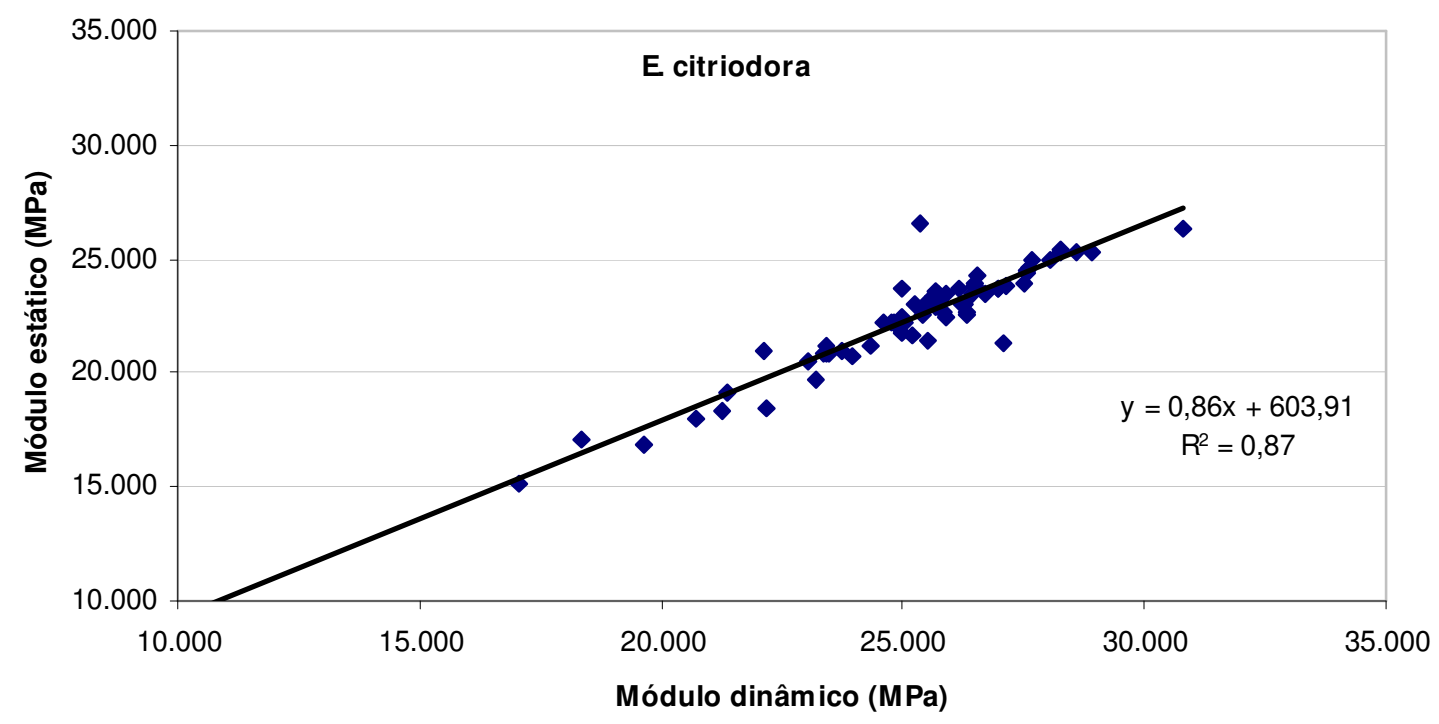

FIGURA 2. Correlação entre os módulos de elasticidade dinâmico e estático para a madeira de $E$. citriodora. 


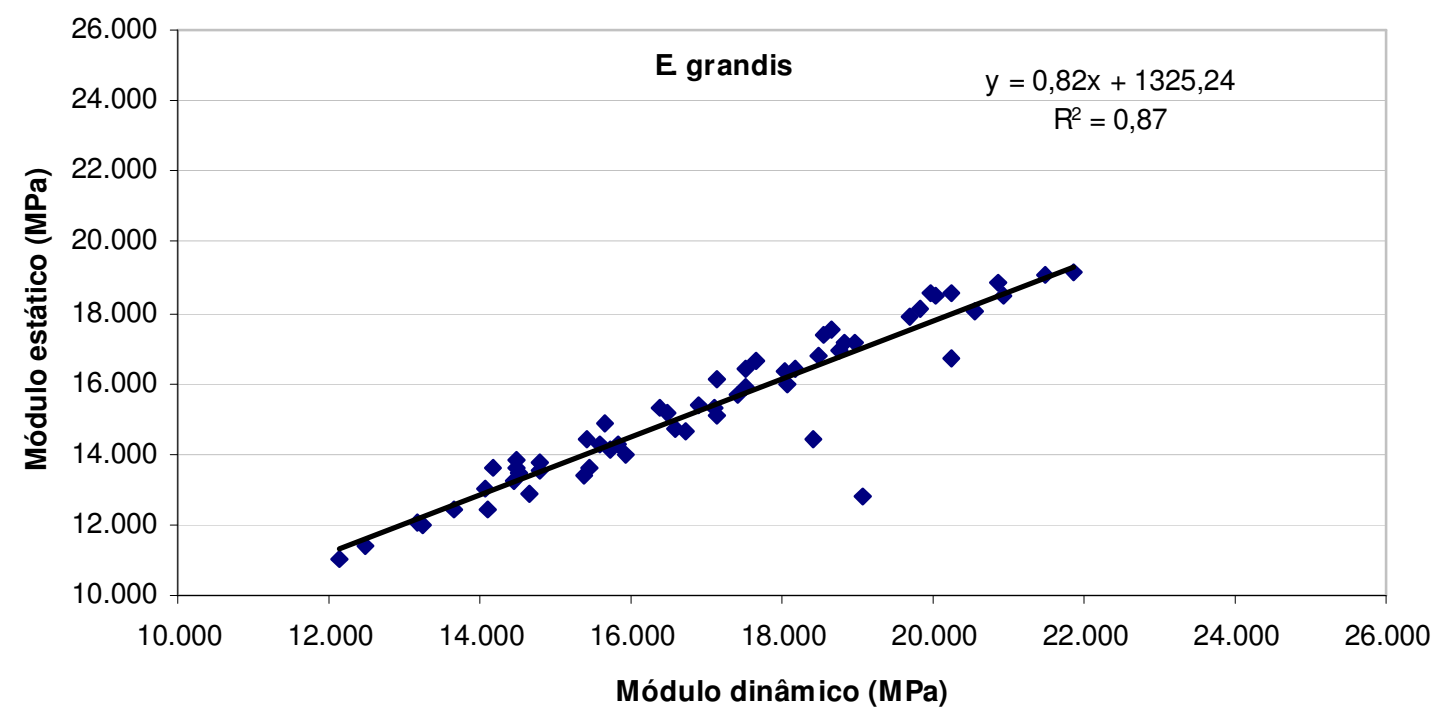

FIGURA 3. Correlação entre os módulos de elasticidade dinâmico e estático para a madeira de $E$. grandis.

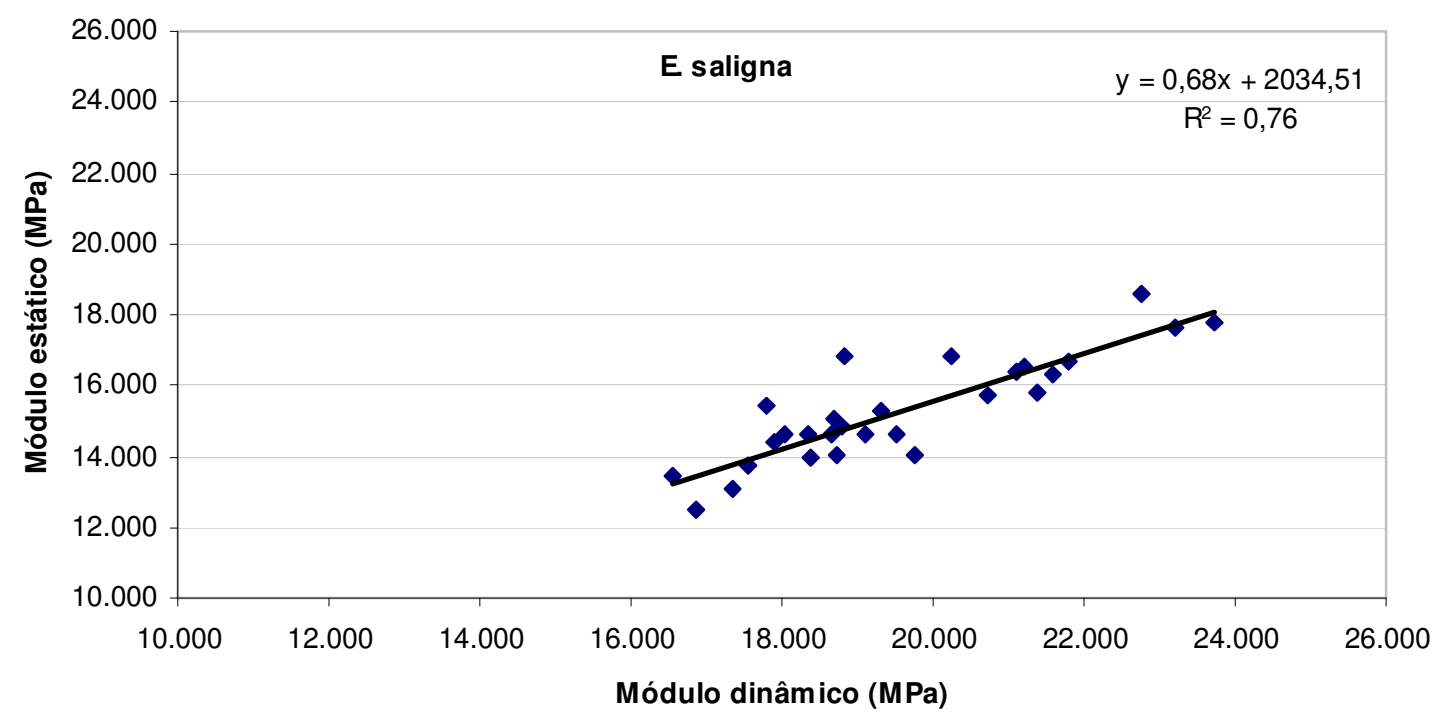

FIGURA 4.Correlação entre os módulos de elasticidade dinâmico e estático para a madeira de $E$. saligna.

Assim, quanto menor a freqüência de excitação do material, maior seria a proximidade entre os resultados obtidos em ensaios dinâmicos e seus correspondentes estáticos. Isso explicaria os melhores resultados obtidos nos ensaios de vibração transversal (frequiências de excitação da ordem de 400 a $500 \mathrm{~Hz}$ ), quando comparados com aqueles do método do ultra-som (freqüências de excitação da ordem de $45 \mathrm{kHz}$ ), por exemplo.

Numa análise global das espécies estudadas, verifica-se que, para os eucaliptos citriodora e grandis, o módulo de elasticidade dinâmico foi cerca de $12 \%$ maior que o estático. Para o eucalipto saligna, o módulo dinâmico foi $28 \%$ superior ao correspondente estático. 
Analisando-se o mesmo quesito dentro de cada espécie, verificou-se, com os diagramas apresentados, tendência de aumento da diferença entre os módulos estáticos e dinâmicos com o aumento do módulo de elasticidade.

Pelo equacionamento teórico do fenômeno [eq.(3)], nota-se que, em se mantendo constantes as demais variáveis, módulos de elasticidade mais elevados estão associados a maiores freqüências de vibração, fato esse comprovado no programa experimental. Assim, dentro de um lote homogêneo (dentro de uma espécie, no caso) para os exemplares com maior módulo de elasticidade, por terem eles maiores freqüências de vibração natural, maior seria a distância existente entre o resultado dinâmico e o estático, numa extensão do raciocínio geral, já apresentado.

\section{CONCLUSÕES}

O método da vibração transversal revela-se como importante ferramenta para inferência nãodestrutiva do módulo de elasticidade da madeira.

Os coeficientes de determinação das regressões realizadas entre os módulos de elasticidade dinâmicos e estáticos foram sempre superiores a $76 \%$, atingindo o valor máximo de $87 \%$ para a madeira de E. grandis. Essas correlações podem ser consideradas altamente significativas.

Os valores do módulo de elasticidade dinâmico, obtidos com uso do método da vibração transversal, foram, no geral, $12 \%$ superiores àqueles dos ensaios convencionais destrutivos de flexão para as madeiras de E. citriodora, E. grandis. Para o E. saligna, os módulos de elasticidade dinâmicos foram cerca de $28 \%$ superiores aos correspondentes estáticos.

Dentro de um lote homogêneo, a diferença entre os resultados de módulos de elasticidade estático e dinâmico aumenta na medida em que aumenta o módulo de elasticidade do exemplar estudado.

\section{REFERÊNCIAS}

ASSOCIAÇÃO BRASILEIRA DE ENSAIOS NÃO-DESTRUTIVOS. Disponível em: $<$ http://www.abende.org.br/analise_vibracoes.html> Acesso em: 15 set. 2002

ASSOCIAÇÃO BRASILEIRA DE NORMAS TÉCNICAS. NBR 7190: Projeto de estruturas de madeira. Rio de Janeiro, 1997. 107 p.

BODIG, J.; JAYNE, B.A. Mechanics of wood and wood composites. Malabar: Krieger, 1993. 712 p.

BUCUR, V. Acoustics of wood. Boca Raton: CRC Press, 1995. 284 p.

BURDZIK, W.M.G.; NKWERA, P.D. Transverse vibration tests for prediction of stiffness and strength properties of full size Eucalyptus grandis. Forest Products Journal, Madison, v.52, n.6, p.63-7, 2002.

CALIL JÚNIOR, C.; MINÁ, A.J.S. Vibração transversal: um método eficiente para classificação de peças estruturais de madeira. Revista Brasileira de Engenharia Agrícola e Ambiental, Campina Grande, v.7, n.2, p.335-8, 2003.

CENTRE DE COOPÉRATION INTERNATIONALE EN RECHERCHE AGRONOMIQUE POUR LE DÉVELOPPEMENT - CIRAD. Foret - Programme Bois - Documentos técnicos de divulgação do BING France, 1998. 10 p.

GOUGH, W.; RICHARDS,. J.P.G.; WILLIAMS, R.P. Vibrations and waves. $2^{\text {nd }}$ ed. London: Prentice Hall, 1983. 249 p. 
HEARMON, R.F.S. An introduction to applied anisotropic elasticity. Oxford: Oxford University Press, 1961. $136 \mathrm{p}$.

HEARMON, R.F.S. Theory of the vibration testing of wood. Forest Products Journal, Madison, v.16, n.8, p.29-40, 1966.

PERSTORPER, M. Dynamic modal tests of timber evaluation according to the Euler and Timoshenko teories. In: INTERNATIONAL SYMPOSIUM ON NONDESTRUCTIVE TESTING OF WOOD, 9., 1993, Pullman. Proceedings ... Pullman: Washington State University, 1993. p.45-54.

ROSS, R.J.; PELLERIN, R.F Nondestructive testing for assessing wood members in structures. Madison: U.S. Department of Agriculture, Forest Service, Forest Products Laboratory, 1994. 40 p. (General Technical Report FPL-GTR 70)

SNOWDON, J.C. Vibration and shock in damped mechanical systems. New York: John Wiley and Sons, 1968. 486 p.

WANG, X.; ROSS, R.J; BRASHAW, B.K.; ERICKSON, J.R.; FORSMAN, J.W.; PELLERIN, R. Diameter effect on stress wave evaluation of modulus of elasticity of logs. In: INTERNATIONAL SYMPOSIUM ON NONDESTRUCTIVE TESTNG OF WOOD, 2002, Berkeley. Proceedings... Madison: FPS, 2002. p.149-56. 